\title{
Radiological signs of pulmonary congestion do not predict failed spontaneous breathing trial
}

\author{
ACP Antonio ${ }^{1,2,3^{*}}$, AP Zanardo ${ }^{3}$, PS Castro ${ }^{2,3}$, MB Gazzana ${ }^{2,3}$, C Teixeira ${ }^{3}$, M Knorst $^{1}$ \\ From ESICM LIVES 2015 \\ Berlin, Germany. 3-7 October 2015
}

\section{Introduction}

Both delayed and premature liberation from mechanical ventilation (MV) are associated with increased morbimortality. Positive pressure ventilation exerts beneficial effects in individuals with cardiogenic pulmonary edema; inspiratory fall in intra-thoracic pressure during spontaneous breathing trial (SBT), in its turn, may precipitate cardiac dysfunction through abrupt increase in venous return and in left ventricular afterload.

\section{Objectives}

Determine the impact of radiological signs of pulmonary congestion prior to submission to SBT on weaning outcomes in a mixed ICU population.

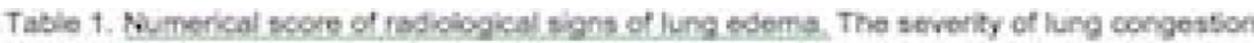 or edema acoording to ractiological scoce (F.S) chteria was determined as follows: RS of 0-1 for a normat chest $X$-ray. $2-4$ - tor interstival lung eongeston, and f-S values of $5-6,7-8$ and 0-10 signified mil, moderate and severe alvoolar edemas, respectively.}

\begin{tabular}{|c|c|}
\hline Sign & value \\
\hline Lurg vessele fedistrbubon- no & a \\
\hline 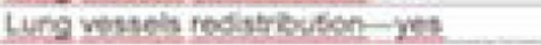 & 1 \\
\hline Woth of cardiac s thouotin $(60 \%)$ - no & 0 \\
\hline Width of cardiac sinouette (both) - yes & $t$ \\
\hline Peribrenchial touffing-no & 0 \\
\hline Phribronchigt culling - yet & 1 \\
\hline New pleural effusion - no & 0 \\
\hline Antw pleural eftusion - unilateral & $t$ \\
\hline Now pleural oftusion - bulaternl & 2 \\
\hline \multicolumn{2}{|l|}{ Kerity A crland B orland C line } \\
\hline Nonen & $\theta$ \\
\hline Ungertain & 1 \\
\hline Detinite & 2 \\
\hline No lyng opasty. & 0 \\
\hline Lung spacity & 1 \\
\hline Lung ground gltis opaciy. & 2 \\
\hline Lung batwing ederna & 3 \\
\hline
\end{tabular}

Figure 1

'Universidade Federal do Rio Grande do Sul, Programa de Pós-Graduação

em Ciências Pneumológicas, Porto Alegre, Brazil

Full list of author information is available at the end of the article

C 2015 Antonio et al.; This is an Open Access article distributed under the terms of the Creative Commons Attribution License (http:// creativecommons.org/licenses/by/4.0), which permits unrestricted use, distribution, and reproduction in any medium, provided the original work is properly cited. 


\section{Methods}

A prospective, observational study in an adult medicalsurgical ICU. All enrolled patients met eligibility criteria for weaning from MV. Traqueostomized subjects were excluded. The primary end point was SBT failure, defined as inability to tolerate a $\mathrm{T}$-piece trial during 30 to 120 minutes, in which case patient was not extubated. An attending radiologist applied a radiological score (RS)

\section{Results}

There was a total of 170 SBTs procedures; SBT failure eventuated in $28(16.4 \%)$. Nineteen patients $(11.2 \%)$ had systolic heart failure (ejection fraction < 35\%), $4(2.4 \%)$ had chronic obstructive pulmonary disease (COPD) and $31(18.2 \%)$ had been intubated due to respiratory sepsis. One hundred thirty-three patients $(78.3 \%)$ were extubated at first attempt. RS was similar between SBT failure and success subjects (median $3[2-4]$ vs $3[2-4], \mathrm{p}=$ 0.146 ), which means only intersticial lung congestion for both groups. Receiver operating characteristic (ROC) curves analysis demonstrated fail accuracy (area under curve $[\mathrm{AUC}]=0.58$ ) of CXRs prior to T-piece trial for discrimination between SBT failure and success individuals. There was no correlation between fluid balance in the 48 hours preceding SBT and RS ( $\rho=-0.13$ ).

\section{Conclusions}

Radiological findings of pulmonary congestion should not delay SBT indication since they did not predict greater probability of SBT failure in medical-surgical critically ill population.

\section{Authors' details}

'Universidade Federal do Rio Grande do Sul, Programa de Pós-Graduação em Ciências Pneumológicas, Porto Alegre, Brazil. ${ }^{2}$ Hospital de Clínicas de Porto Alegre, Porto Alegre, Brazil. ${ }^{3}$ Hospital Moinhos de Vento, Porto Alegre, Brazil.

Published: 1 October 2015

\section{Reference}

1. Shochat M, Shotan A, Trachtengerts $V$, Blondheim DS, Kazatsker M, Gurovich V, Asif A, Shochat I, Rozenman Y, Meisel SR: A novel radiological score to assess lung fluid content during evolving acute heartfailure in the course of acute myocardial infarction. Acute Card Care 2011, 13(2):81-6, Jun;

doi:10.1186/2197-425X-3-S1-A1007

Cite this article as: Antonio et al:: Radiological signs of pulmonary congestion do not predict failed spontaneous breathing trial. Intensive Care Medicine Experimental 2015 3(Suppl 1):A1007.

\section{Submit your manuscript to a SpringerOpen ${ }^{\mathcal{O}}$ journal and benefit from:}

- Convenient online submission

- Rigorous peer review

- Immediate publication on acceptance

- Open access: articles freely available online

- High visibility within the field

- Retaining the copyright to your article

Submit your next manuscript at springeropen.com 